\title{
Um modelo multiobjetivo de otimização aplicado ao processo de orçamento de capital
}

\section{A multicriteria optimization model applied to the capital budgeting process}

\author{
Eder Oliveira Abensur ${ }^{1}$
}

\begin{abstract}
Resumo: O processo de orçamento de capital envolve a análise e seleção de projetos de longo prazo de maturação. Essas decisões de investimento são tradicionalmente feitas pela aplicação simultânea de vários métodos financeiros com uso de fluxo de caixa descontado como, por exemplo, o Valor Presente Líquido (VPL) e a Taxa Interna de Retorno (TIR). Apesar da longa e ampla disseminação dessas técnicas, são notórios os problemas de avaliação especialmente quando consideradas funções mono-objetivas e projetos mutuamente excludentes. Em se tratando de decisões financeiras, parece ilusório falar em otimização sem considerar múltiplos objetivos e atributos. O objetivo deste trabalho é propor um modelo matemático multiobjetivo prático que auxilie na seleção de projetos de investimento submetidos simultaneamente a vários indicadores de desempenho que incorpora uma nova medida de risco $\left(\mathrm{GA}_{\mathrm{FT}}\right)$. Os testes foram feitos sobre uma amostra de quarenta e cinco projetos e os resultados demonstram que o modelo proposto é uma ferramenta gerencial prática e promissora.
\end{abstract}

Palavras-chave: Análise de investimentos. Otimização multicritério. Programação linear inteira.

\begin{abstract}
The capital budgeting process involves the analysis and selection of projects committed over long periods of time. These investment decisions are traditionally made by the simultaneous application of various financial techniques using discounted cash flow, such as the Net Present Value (NPV) and Internal Rate of Return (IRR). Despite the long-term and wide dissemination of these techniques, there are major problems of inconsistency especially in mono-criterion functions and mutually exclusive projects. When dealing with financial decisions, it seems illusory to address optimization without taking multiple objectives and attributes into account. The objective of this paper is to present a mathematical model that allows the multi-criteria selection of investment projects submitted to various financial indicators; the mathematical model incorporates a new measure of risk (GAFT). Forty five projects were tested and the results show that the proposed model is a practical and promising decision-making tool.
\end{abstract}

Keywords: Investment analysis. Multi-criteria optimization. Integer linear programming.

\section{Introdução}

A análise das propostas de orçamentos de capital ou de projetos de investimentos é uma crucial tarefa realizada pelas empresas para obtenção de crescimento ou manutenção da sobrevivência nos mercados em que atuam. Ao longo do tempo, diversas técnicas de análise foram desenvolvidas para dar suporte às decisões relacionadas à seleção, priorização e alocação de recursos limitados em projetos.

É comum, principalmente em grandes empresas, a concorrência dos projetos de investimento entre si pela alocação dos limitados recursos colocados à disposição para esta finalidade. Neste contexto, cabe aos gestores selecionar, dentre as várias alternativas apresentadas, aquelas que irão compor o portfólio de projetos do período em análise. Na prática, o racionamento de capital é imposto pelas empresas para evitar níveis desnecessários e excessivos de novos financiamentos.

A Companhia Vale do Rio Doce divulgou vinte e um projetos, selecionados em 2010, com orçamento de US\$ 6,9 bilhões divididos em minerais ferrosos, minerais não ferrosos, logística, carvão e energia (COMPANHIA..., 2011a). O orçamento de capital representou quase $40 \%$ do lucro líquido da empresa em 2010 (COMPANHIA..., 2011b). Estes números dão uma ideia da dimensão e da importância deste tema para a gestão empresarial.

De forma geral, as empresas aplicam múltiplos métodos para a gestão do portfólio de seus projetos, tais como: (i) métodos financeiros (77,3\%); (ii) métodos de estratégia do negócio (64,8\%); (iii) diagramas de bolhas (40,6\%); (iv) scoring models (37,9\%);

\footnotetext{
${ }^{1}$ Centro de Engenharia, Modelagem e Ciências Sociais Aplicadas - CECS, Universidade Federal do ABC - UFABC,

CEP 09210-170, Santo André, SP, Brasil, e-mail: eder.abensur@ufabc.edu.br
} 
e (v) checklists (20,9\%). Conforme apresentado, as técnicas financeiras são as mais empregadas (COOPER; EDGETT; KLEINSCHMIDT, 1999).

No Brasil, Eid Júnior (1996) analisou as técnicas de análise de investimentos utilizadas pelas empresas brasileiras e concluiu que: (i) $22 \%$ usam preferencialmente o VPL; (ii) $23 \%$ usam preferencialmente a $T I R$; (iii) $25 \%$ usam preferencialmente o pay-back $(P B)$; (iv) $6 \%$ usam $V P L+T I R$; (v) $3 \%$ usam $V P L+P B$; (vi) $13 \%$ usam $T I R+P B ;$ e (vii) $10 \%$ usam todas. De acordo com a pesquisa, praticamente todas as empresas utilizam-se de mais de uma técnica ao mesmo tempo. O uso de múltiplas técnicas de análise também foi confirmado no mercado americano por Brealey, Myers e Allen (2008).

Há varias formas de classificação dos projetos de investimento, mas uma forma usual divide-os em: (i) independentes, quando a decisão de investir em um não impede a seleção de outros; (ii) dependentes (contingentes), quando a escolha de um projeto está condicionada a seleção de um ou de outros projetos; e (iii) mutuamente excludentes, quando a seleção de um projeto impede a escolha de outros. Os projetos podem estar inseridos num contexto de restrição de capital, quando há um limite de investimento para os projetos, ou sem restrição de capital. É comum, além de ser gerencialmente mais relevante, existir a combinação das três primeiras alternativas nas avaliações de propostas, ou seja, projetos independentes, dependentes e/ou mutuamente excludentes com restrição de capital. Vale ressaltar que a restrição de capital, por si só, já representa um tipo de dependência entre todos os projetos avaliados.

Como regra geral, os métodos financeiros baseiam-se na estimativa dos fluxos de caixa incrementais dos projetos avaliados. Consideram-se como incrementais aqueles após o desconto de impostos e da depreciação. Tradicionalmente, os seis métodos mais empregados para classificar projetos e para decidir se devem ou não ser aceitos para inclusão no orçamento de capital são: (i) pay-back $(P B)$; (ii) pay-back descontado $(P B D)$; (iii) valor presente líquido $(V P L)$; (iv) índice de lucratividade $(I L)$; (v) taxa interna de retorno (TIR); e (vi) taxa interna de retorno modificada (MTIR) (BRIGHAM; GAPENSKI; EHRHARDT, 2001).

Com o intuito de aumentar o grau de conhecimento dos projetos analisados e a confiabilidade na tomada de decisão, vários métodos são usados na avaliação das propostas. Além da dificuldade operacional de se avaliar dezenas de projetos simultaneamente, muitas vezes os projetos apresentam sinais contraditórios nos indicadores analisados, especialmente, em se tratando de projetos mutuamente excludentes (BRIGHAM; GAPENSKI; EHRHARDT, 2001).
As decisões financeiras encontram-se geralmente inseridas num contexto de otimização. No longo prazo, estão as decisões relacionadas com a alocação ótima dos recursos e a estrutura ótima de capital. No curto prazo, estão as decisões ligadas à otimização de ações, gerenciamento de caixa, passivo circulante etc. As possibilidades de otimização dessas decisões conduziram os pesquisadores a fazerem propostas de técnicas de pesquisa operacional para resolução de tais problemas.

Com o tempo, os métodos tradicionalmente aplicados ao suporte de decisões financeiras foram questionados quanto a sua capacidade de tratar eficazmente problemas que aumentavam em grau de complexidade, número de variáveis envolvidas e que possuíam não apenas um, mas múltiplos objetivos de otimização. Os métodos multicriteriais incorporaram uma perspectiva multidimensional para o tratamento de situações financeiras ao levarem em consideração vários objetivos organizacionais.

O propósito deste trabalho foi investigar a viabilidade de aplicação de um modelo prático multiobjetivo como suporte às decisões de orçamento de capital de projetos de diferentes naturezas submetidos a múltiplos indicadores de desempenho e com restrição orçamentária no início da análise dos projetos. Para tanto, os seguintes objetivos foram definidos: (i) identificar as variáveis relevantes para o método; (ii) apresentar uma seleção e classificação de projetos conforme os resultados da aplicação do modelo; e (iii) avaliar a eficiência do modelo proposto perante outras técnicas usualmente aplicadas.

Uma revisão bibliográfica definiu os métodos de análise de investimentos mais praticados, suas vantagens e desvantagens em relação à natureza dos projetos analisados. Esta revisão serviu, também, para definição dos indicadores a serem empregados na função objetivo do modelo proposto e na forma de comparação dos resultados obtidos.

Um obstáculo encontrado no estudo foi a obtenção de dados para comparação entre os métodos tradicionais e proposto. Os dados de projetos reais publicados na literatura eram insuficientes para os testes, principalmente, porque não permitiam a reconstituição do fluxo de caixa. Decidiu-se, então, pela coleta de dados de exemplos teóricos publicados. Para garantir um relativo rigor estatístico, a amostra foi constituída por seleção a esmo, evitando-se, assim, a influência de alguma característica dos elementos da população ou de erro de julgamento do amostrador (COSTA NETO, 2000). Esta opção em nada afetou o desempenho ou a qualidade dos resultados obtidos.

Quarenta e cinco (45) projetos de investimento foram levantados na literatura especializada e divididos em 16 grupos formando uma amostra diversificada e heterogênea em termos de: (i) magnitude dos investimentos iniciais; (ii) vidas úteis; (iii) taxas 
mínimas de atratividade (TMA); (iv) natureza dos projetos (independentes, dependentes, mutuamente excludentes); e (v) indicadores de desempenho (rentabilidade, liquidez, risco). Os projetos foram selecionados em função do modelo matemático proposto e comparados com a seleção oferecida pelos métodos mais recomendados de análise de investimentos. Para realização das simulações, foi desenvolvido um aplicativo baseado em planilha Excel e no pacote de otimização Solver do Microsoft Excel ${ }^{\circledR}$.

$\mathrm{O}$ trabalho foi estruturado de tal forma que as primeiras seções apresentam os fundamentos para a composição do modelo matemático proposto. As seções 2 e 3 descrevem, respectivamente, os métodos de análise de investimento e a justificativa de uso dos atributos de rentabilidade, liquidez e risco criados para as análises. A seção 4 explica o método de busca empregado. A seção 5 mostra a formulação do modelo proposto. Na seção 6, os testes aplicados são caracterizados e os resultados apresentados. A seção 7 mostra as conclusões do estudo.

\section{Métodos tradicionais de análise de investimentos}

Os métodos tradicionais de análise de investimentos partem do fluxo de caixa descontado (FCD) para suas aplicações. O objetivo do fluxo de caixa é projetar o resultado futuro da empresa fruto de suas atividades atuais ou o valor agregado por um novo projeto de investimentos. Eles refletem o resultado líquido das entradas menos as saídas previstas para o projeto durante a sua vida útil. Fluxos passados não são considerados para a análise. O fluxo de caixa líquido (FCL) resultante já desconta os impactos fiscais (impostos) e de depreciação.

Conforme sua natureza, os FCLs podem ser divididos em: (i) certos ou determinísticos, quando há uma boa previsibilidade e confiabilidade na formação e constituição do fluxo previsto do projeto; ou (ii) incertos e estocásticos, quando há um risco assumido na formação do fluxo. A maioria das aplicações e análises praticadas em âmbito gerencial está contida no primeiro grupo. As aplicações do segundo grupo demandam um maior grau de sofisticação com emprego de técnicas como Teoria de Opções Reais, Árvores Binomiais, Simulação de Monte Carlo etc.

De forma geral, um fluxo de caixa é composto dos seguintes componentes: (i) investimento ou desembolso inicial $(D I)$, que envolve as aquisições de ativos para início do projeto; (ii) fluxo de caixa líquido, resultado do incremento das entradas menos saídas previstas do projeto; (iii) taxa mínima de atratividade (TMA); (iv) vida útil e (v) valor residual, que envolve um impacto monetário no caixa do projeto ao final de sua vida útil estimada.

\subsection{Valor Presente Líquido (VPL) e Índice de Lucratividade (IL)}

Pelo método do VPL, os fluxos de caixa do projeto são convertidos ao valor presente (momento inicial $t_{0}$ ) por meio da aplicação de uma taxa de desconto determinada que pode corresponder ao custo de capital da empresa ou à taxa mínima de atratividade (TMA) aceitável em face do risco envolvido. Embora qualquer período da vida útil do projeto possa ser escolhido para avaliação dos resultados, o momento inicial $t_{0}$ é geralmente adotado para este propósito, pois é no momento presente que há mais condições dos gestores de avaliarem os valores monetários resultantes da aplicação do método. Seriam aceitos todos os projetos que apresentassem VPL positivo. As premissas do VPL são: (i) conhecimento do custo de capital ou da TMA para os projetos avaliados; e (ii) definição das vidas úteis dos projetos.

As principais desvantagens da aplicação do $V P L$ são: (i) definição e confiabilidade da TMA e dos fluxos de caixa futuros do projeto, que nem sempre é uma tarefa simples; (ii) simplificação da realidade pela suposição que todas as entradas e saídas do fluxo de caixa são aplicados à mesma TMA; (iii) o resultado do método é um valor absoluto expresso em termos monetários, acarretando numa perda de referência do desembolso inicial investido no projeto; e (iv) é considerado inadequado para comparações de projetos mutuamente excludentes com vidas úteis desiguais, sendo mais apropriado para projetos independentes (CASAROTTO FILHO; KOPITTKE, 2010; HIRSCHFELD, 2009). A Fórmula geral do método VPL é expressa a seguir (Equação 1).

$$
V_{P L}=\sum_{j=1}^{N} \frac{P_{j}}{(1+i)^{j}}-D I
$$

em que: $i=$ custo de capital ou taxa mínima de atratividade (TMA); $P_{j}=$ fluxo de caixa líquido na data $j ; D I=$ desembolso inicial ou investimento inicial; $N=\mathrm{n}^{\mathrm{o}}$ de períodos.

Quando o método do VPL é aplicado a projetos de diferentes grandezas, ele dá como resultado valores absolutos, dificultando sua classificação em função dos seus retornos.

O índice de lucratividade (IL) ou de rentabilidade foi criado para permitir a classificação de projetos em função do retorno. O resultado do Valor Presente do fluxo é dividido pelo seu respectivo desembolso inicial, propiciando um valor relativo comparável com diferentes projetos de diferentes vidas úteis. A fórmula geral do método é mostrada a seguir (Equação 2).

$$
I L=\frac{\sum_{j=1}^{N} \frac{P_{j}}{(1+i)^{j}}}{D I}
$$


Um $I L=1$ representa a obtenção da taxa mínima de retorno exigida durante o período de vida útil do projeto. $\mathrm{O}$ excesso em relação a $I L=1$ (ex: $1,03-1=0,03$ ) representa o retorno total obtido além do mínimo exigido. Seriam aceitos os projetos com $I L>1$.

As principais desvantagens do método são: (i) definição e confiabilidade da TMA e dos fluxos de caixa futuros do projeto; (ii) simplificação da realidade pela suposição de que todas as entradas e saídas do fluxo de caixa são aplicadas à mesma TMA e (iii) para projetos mutuamente excludentes, não há garantias de que os projetos com maior $I L$ sejam os melhores (ROSS; WESTERFIELD; JAFFE, 2002).

Conforme Ross, Westerfield e Jaffe (2002), em situações de racionamento de capital em um único período, os projetos devem ser classificados de acordo com o $I L$ ao invés do VPL. Portanto, o método do $I L$ possui especial emprego na ordenação de projetos em situações de restrição de capital por maximizar a eficiência da rentabilidade do projeto por unidade monetária investida (SAMANEZ, 2009).

\subsection{Taxa Interna de Retorno (TIR) e Taxa Interna de Retorno Modificada (MTIR)}

A Taxa Interna de Retorno (TIR) é a taxa de desconto $(i)$ que iguala o valor presente das entradas líquidas de caixa ao valor presente dos desembolsos relativos ao investimento. A denominação de taxa interna é oriunda do fato de que o valor é calculado, exclusivamente, em função dos fluxos de caixa do projeto e independe dos juros de mercado. A TIR deve ser comparada a uma taxa mínima estipulada, por exemplo, ao custo de capital da empresa ou a $T M A$. Se a TIR for maior ou igual à taxa mínima, o projeto poderá ser aprovado. Se a $T I R$ for inferior à taxa mínima, o projeto deve ser rejeitado. A expressão geral de formação da $T I R$ é apresentada a seguir (Equação 3).

$$
\sum_{j=1}^{N} \frac{P_{j}}{(1+i)^{j}}-D I=0
$$

Ao longo do tempo, os gestores empresariais demonstraram simpatia e preferência por este método, pois ele resume a avaliação do projeto a um percentual que pode ser comparado aos outros analisados. No entanto, há vários problemas de ordem operacional e conceitual inerentes a sua aplicação como:

- Alguns fluxos de caixa não têm TIR

A TIR é a taxa que torna nulo o valor presente líquido $(V P L)$ do fluxo de caixa. Como o fluxo de caixa pode ter $n$ períodos de apuração, a expressão matemática da $T I R$ recai na solução de uma equação matemática de grau $n$ ou de um polinômio de grau $n$ com $n$ possíveis raízes.

- Fluxos de caixa com múltiplas raízes

É uma possibilidade inerente a uma equação de grau $n$.

- Fluxos de caixa de projetos mutuamente excludentes

Geralmente, o VPL e a TIR apontam para a mesma decisão de aceitar ou rejeitar um projeto, quando estes são independentes. Entretanto, quando analisados projetos mutuamente excludentes, especialmente de desembolsos iniciais diferentes, a seleção pela $T I R$ pode levar a decisões inconsistentes.

- Reaplicação do investimento

O método pressupõe que todo o fluxo de caixa gerado é aplicado à TIR. Esta suposição é de difícil aceitação, principalmente em projetos que apuram valores de TIR muito acima das taxas praticadas pelo mercado.

A taxa interna de retorno modificada (MTIR) foi concebida para corrigir os problemas de raízes múltiplas ou inexistentes e da taxa de reaplicação do investimento da TIR. Os fluxos de caixa são levados ao valor futuro pelo custo de capital ou pela TMA. O valor futuro final é comparado ao desembolso inicial para determinação da taxa de equilíbrio $(M T I R)$. Em termos práticos, a MTIR oferece valores inferiores e mais realistas do que a TIR. A fórmula geral de formação da MTIR é expressa a seguir (Equação 4).

$$
\frac{\sum_{j=1}^{N-j} P_{j}(1+T M A)^{N-j}}{(1+M T I R)^{N}}-D I=0
$$

\subsection{Payback (PB) e Payback Descontado (PBD)}

O método do Payback é considerado como o primeiro método formal utilizado para avaliar projetos de orçamento de capital (BRIGHAM; GAPENSKI; EHRHARDT, 2001). Ele tem origem numa concepção simples que determina o tempo necessário ou o prazo de retorno para recuperar os recursos investidos $(D I)$ em um período. O cálculo do prazo de retorno é feito da seguinte forma: (i) se as entradas líquidas de caixa forem constantes, bastará dividir o investimento inicial $(D I)$ pelas entradas anuais de caixa; ou (ii) se as entradas líquidas forem diferentes, elas deverão ser acumuladas até recuperar o valor investido, apurando-se o prazo de retorno.

De forma geral, seriam aceitos todos os projetos que apresentassem um payback inferior a um prazo limite e arbitrário estabelecido pela empresa. As principais desvantagens apresentadas pelo método são: (i) não reconhece as entradas de caixa previstas para ocorrerem após a recuperação do investimento, ou seja, depois do prazo máximo estabelecido; (ii) não 
avalia adequadamente o valor do dinheiro no tempo; e (iii) é influenciado pela distribuição dos fluxos de caixa dentro do período de payback, ou seja, o método privilegia os projetos cujas entradas maiores aconteçam mais cedo, mas que não são, necessariamente, os de melhor rentabilidade.

Com o intuito de corrigir as falhas do método de payback tradicional, criou-se uma variante denominada de payback descontado $(P B D)$. Este método determina o tempo necessário para recuperar os recursos investidos em um período, considerando os fluxos de caixa descontados. O PBD corrige o fato de o payback clássico desconsiderar o valor do dinheiro no tempo. Entretanto, ele possui a mesma deficiência de desconsiderar as entradas de caixa previstas para ocorrerem depois da recuperação do investimento. A fórmula geral do $P B D$ é apresentada a seguir (Equação 5).

$$
D I=\sum_{j=1}^{N} \frac{P_{j}}{(1+i)^{j}}
$$

Apesar das limitações expostas pelos métodos do $P B$ e do $P B D$, eles são usados em muitas decisões de investimento que compreendem, especialmente, curtos espaços de tempo ou que envolvem montantes de pequeno porte (HIRSCHFELD, 2009; ROSS; WESTERFIELD; JAFFE, 2002). Por refletirem a velocidade de recuperação do capital investido, eles também são considerados como medidas de liquidez. Devido a sua simplicidade, os métodos de $P B$ e $P B D$ são usados de forma complementar ou como filtros na tomada de numerosas decisões de investimento (BREALEY; MYERS; ALLEN, 2008; ROSS; WESTERFIELD; JAFFE, 2002).

Os métodos apresentados são os mais destacados na literatura e amplamente usados no ambiente empresarial. Academicamente, há uma preferência pelo método do $V P L$, entretanto as mesmas fontes reforçam que, diante do racionamento de capital, o método do $I L$ é o mais recomendado. O critério da TIR ou da MTIR não é recomendado para hierarquizar projetos em situações de restrição de capital. A Tabela 1 resume as recomendações de métodos de análise de investimentos conforme as naturezas e as características dos projetos avaliados. As recomendações não impedem a aplicação dos demais métodos.

\section{Medida de risco dos projetos de investimentos}

Esta seção apresenta e discute várias medidas de risco aplicáveis à seleção de projetos.

O risco pode ser compreendido como a probabilidade de insucesso. Uma condição de risco pode ser entendida como uma situação em que probabilidades objetivas podem ser associadas aos resultados do projeto. Ao contrário, numa situação de incerteza, não há possibilidades de se associar uma distribuição de probabilidade de ocorrência ou somente se pode associar uma distribuição subjetiva (CASAROTTO FILHO; KOPITTKE, 2010).

No Brasil, a NBR ISO 31000 (ASSOCIAÇÃO..., 2009) estabelece um processo de gestão de risco composto por sete etapas que incluem a identificação (fonte dos riscos), análise (relação causa-consequência), avaliação (decisão baseada no risco) e tratamento dos riscos (ações para enfrentar os riscos identificados). A norma ainda estabelece que devem ser utilizadas as melhores informações disponíveis para a avaliação e priorização dos riscos.

$\mathrm{Na}$ forma de fluxo de caixa convencional, alguns fatores de risco podem ser identificados para os projetos de investimento, tais como: (i) flutuação ou volatilidade da taxa de juros ou da TMA; (ii) risco de estimativa da vida útil; (iii) risco da data de ocorrência do fluxo de caixa; e (iv) risco sobre o valor estimado do fluxo de caixa.

A partir de princípios estatísticos (covariância, variância, média, desvio padrão) e probabilísticos (retorno esperado de uma variável aleatória), Markowitz (1952) formulou o risco de uma carteira

Tabela 1. Recomendações de métodos de análise de investimentos para seleção de projetos.

\begin{tabular}{cclc}
\hline Natureza dos projetos & Capital racionado $^{\mathbf{1}}$ & Vidas úteis & Métodos mais recomendados \\
\hline \multirow{4}{*}{ Independentes } & $\mathrm{Sim}$ & Distintas & $I L$ \\
& $\mathrm{Sim}$ & Equivalentes & $I L / V P L$ \\
& $\mathrm{Não}$ & Distintas & $I L / V P L^{2}$ \\
& $\mathrm{Não}$ & Equivalentes & $V P L$ \\
\hline \multirow{4}{*}{ Mutuamente excludentes } & $\mathrm{Sim}$ & Distintas & $I L$ \\
& $\mathrm{Sim}$ & Equivalentes & $I L$ \\
& $\mathrm{Não}$ & Distintas & $I L / V P L^{2}$ \\
& $\mathrm{Não}$ & Equivalentes & $V P L$ \\
\hline
\end{tabular}

Fonte: Elaborado pelo autor. Fonte: Brigham, Gapenski e Ehrhardt (2001), Hirschfeld (2009), Ross, Westerfield e Jaffe (2002) e Samanez (2009). ${ }^{1}$ Ocorre uma única vez no início dos projetos. ${ }^{2}$ Com uso do método do mínimo múltiplo comum para equivalência das vidas úteis. 
genérica de ativos admitindo-se que os retornos dos ativos individuais estão relacionados entre si. Markowitz formulou que a variância (ou risco) de um portfolio genérico composto por $n$ ativos depende das variâncias individuais dos títulos e das covariâncias entre os pares de ativos. Para os propósitos dos projetos de investimento analisados neste estudo, dados históricos são praticamente inexistentes especialmente em se tratando de novos projetos.

Sharpe (1964) desenvolveu os fundamentos do modelo de precificação de ativos financeiros (CAPM). Entre as principais conclusões deste modelo, está a definição de uma medida de risco expressa por beta $(\beta)$ que significa a sensibilidade do retorno do título a oscilações de mercado. Entretanto, o CAPM não é diretamente aplicável às decisões de orçamento de capital, pois se baseia num suposto mercado eficiente, que não existe para ativos corporativos reais (não financeiros), como edificações e equipamentos (GITMAN; MADURA, 2003).

Hirschfeld (2009) apresentou o cálculo da probabilidade de um determinado projeto não gerar riqueza, ou seja, do $V P L$ ser negativo $(V P L<0)$. Uma probabilidade de ocorrência é associada a cada entrada do fluxo de caixa e, a partir disso, calcula-se o $V P L$ médio e assume-se a distribuição de probabilidade normal para geração dos desvios padrões e cálculo da probabilidade de inviabilidade do projeto. Apesar da praticidade e da fundamentação estatística, o procedimento apresenta a desvantagem de assumir que a distribuição de probabilidade seja normal para todos os casos. A distribuição normal representa adequadamente muitas distribuições empíricas, mas isto não é verdade para projetos com poucas entradas (JORION, 2003).

$\mathrm{O}$ Value at Risk (VAR) sintetiza a maior (ou pior) perda esperada dentro de determinados período de tempo e intervalo de confiança (JORION, 2003). Uma das formas mais práticas de cálculo do VAR é a forma paramétrica simplificada, que parte da suposição de distribuição normal para estimativa da perda esperada. Assim como na proposta anterior, há a desvantagem conhecida de assumir a distribuição de probabilidade como sendo normal para todas as situações. Além disso, o resultado final é expresso em valores monetários que acarreta uma perda de referência do investimento feito.

A proposta de medida de risco para avaliação dos projetos surge da combinação dos conceitos de análise de sensibilidade de fluxo (HIRSCHFELD, 2009; GITMAN; MADURA, 2003) e da gestão de risco da NBR ISO 31000 (ASSOCIAÇÃO..., 2009). Conforme Eid Júnior (1996), o ajuste de risco mais comum praticado pelas empresas brasileiras é o feito pelo fluxo. Gitman e Madura (2003) esclarecem que nos projetos de orçamento de capital convencionais, o risco vem quase que totalmente das entradas de caixa, porque o investimento inicial geralmente é conhecido com relativa certeza. O histórico brasileiro (BANCO..., 2011) apresenta uma amplitude de 430\% na taxa de juros básica (taxa selic) nos últimos quinze anos demonstrando que as grandes variações nas taxas de juros também representam um importante fator de risco à viabilidade dos negócios.

A proposta de um novo indicador de risco adaptado às características dos fluxos de caixa do processo de orçamento de capital proposto por este estudo foi denominado de grau de alavancagem combinado de fluxo e taxa $\left(G A_{F T}\right)$. Avaliou-se, separadamente, o impacto de um aumento de $10 \%$ nas entradas do fluxo de caixa e na TMA sobre o valor presente do projeto. $\mathrm{O}$ resultado foi medido pelo aumento percentual em relação ao valor presente $(V P)$ inicial. A fórmula geral do grau do $\left(G A_{F T}\right)$ é apresentada a seguir (Equação 6).

$$
G A_{F T}=\left[\begin{array}{l}
\left(1+\left|\frac{\Delta V P(\mathrm{R} \$) \text { devido às entradas }}{V P \text { inicial }(\mathrm{R} \$)}\right|\right) \times \\
\left(1+\left|\frac{\Delta V P(\mathrm{R} \$) \text { devido à taxa }}{V P \text { inicial }(\mathrm{R} \$)}\right|\right)-1
\end{array}\right] \times 100=\Delta \%
$$

O indicador proposto apresenta as seguintes vantagens: (i) incorpora uma avaliação individual de risco dos projetos; (ii) não trabalha com suposições de distribuições de probabilidade; (iii) pode ser obtido com os dados disponíveis do fluxo de caixa; e (iv) a avaliação é feita em mais de uma dimensão. Como desvantagem há a escolha arbitrária do aumento percentual praticado.

\section{A programação linear inteira e a programação de metas}

Um problema de Programação Linear Inteira é uma Programação Linear em que uma ou mais variáveis de decisão são representadas apenas por valores inteiros (LACHTERMACHER, 2002). Eles são de dois tipos: (i) Programação Inteira total em que todas as variáveis de decisão são do tipo inteiro; e (ii) Programação Inteira mista em que apenas uma parte das variáveis é do tipo inteiro.

Uma situação de larga aplicação é quando as variáveis são binárias $(0,1)$. Neste tipo de representação, há a possibilidade de expressar restrições lógicas sofisticadas como, por exemplo, o fato da variável existir ou não diante de certas premissas. O problema clássico de seleção de projetos de investimento recai neste tipo de situação, pois ou o projeto é ou não é selecionado conforme as regras de desempenho estabelecidas.

Os modelos de programação linear, em geral, são baseados na otimização de uma única função objetivo. Há situações em que múltiplos objetivos (conflitantes) podem ser mais adequados. O que a programação de metas faz é buscar uma solução de 
compromisso baseada na importância relativa de cada objetivo (TAHA, 2008).

O modelo básico de programação de metas pode ser entendido considerando-se que $x_{1}, x_{2}, \ldots ., x_{n}$ sejam as variáveis de decisão do problema e que $K$ seja o número de metas. Para cada $x_{j}(j=1,2, \ldots, n)$ e cada $k(k=1,2, \ldots, K)$ tem-se um coeficiente $c_{k}$ da função objetivo e um valor de meta denominado $g_{k}$. Se chamarmos $y_{k}$ de desvio da meta $k$, pode-se defini-lo como (COLIN, 2007; HILLIER; LIEBERMAN, 1988) (Equação 7):

$$
y_{k}=\sum_{j=1}^{n} c_{j k} x_{j}-g_{k}
$$

A função objetivo será a minimização do somatório dos desvios de cada meta (Equação 8).

$$
\operatorname{Min} Z=\sum_{k=1}^{K}\left|y_{k}\right|
$$

A programação linear inteira trabalha com variáveis do tipo $\{0,1\}$ e oferece garantia de solução ótima, ao contrário, de outras técnicas como a programação de metas (COLIN, 2007; HILLIER; LIEBERMAN, 1988; MACEDO, 2005; TAHA, 2008).

\section{$5 \mathrm{O}$ modelo proposto}

O modelo proposto caracteriza-se como multiobjetivo de programação linear inteira (PADOVANI; CARVALHO; MUSCAT, 2010; SOUZA; KLIEMANN NETO; FILOMENA, 2010). Conforme visto na seção 2 , o modelo incorpora o índice de lucratividade $(I L)$ como medida de rentabilidade considerada a mais apropriada para as comparações. Além disso, incluiu-se a medida de liquidez do payback descontado $(P B D)$ e a medida de risco $\left(G A_{F T}\right)$ criada para avaliação dos projetos de investimentos. Outra diferenciação na função objetivo refere-se às relações dos seus componentes. Sendo a função objetivo de maximização, as parcelas com sinais negativos tendem a ser minimizadas, ou seja, menores $P B D$ e $G A_{F T}$.

As restrições dividem-se em: (i) num grupo tradicional de relações de mútua excludência e dependência dos projetos; (ii) numa também tradicional relação de controle do orçamento previsto para os projetos (limite de investimento); e (iii) em relações extras que garantam que os projetos da solução ótima, tenham, respectivamente, MTIR superior a TMA, $I L$ superior a 1 e $P B D$ inferior ou igual à vida útil.

Além disso, as seguintes premissas foram consideradas:

- Todos os projetos começam suas atividades na mesma data inicial;

- Os grupos de projetos são independentes entre si;

- Há grupos com projetos mutuamente excludentes;

- Há projetos independentes;
- Há projetos com relação de dependência;

- A restrição de orçamento de capital ocorre uma única vez na data inicial de análise dos projetos; $\mathrm{e}$

- Assumiu-se, no $2^{\circ}$ estágio do modelo, o mesmo peso $(w)$ para todos os componentes da função objetivo.

Um problema operacional inerente à geração independente de diferentes indicadores está nas suas escalas, pois, embora na mesma unidade (ex: percentual), as grandezas podem estar expressas em valores distantes (ex: dezenas vs. centenas). Em razão disto, o modelo foi dividido em dois estágios: (i) geração dos coeficientes de escala; e (ii) seleção dos projetos. A função do primeiro estágio é determinar os coeficientes que serão empregados na função objetivo do segundo estágio para neutralizar o problema de escala das variáveis de decisão. Os modelos matemáticos propostos são apresentados a seguir (Equação 9).

$$
\begin{aligned}
& Z=f_{1} \sum_{i=1}^{N} I L_{i}+f_{2} \sum_{i=1}^{N} P B D_{i}+f_{3} \sum_{i=1}^{N} G A F T i=0 \\
& \text { s.a. } \\
& f_{i} \geq 1
\end{aligned}
$$

em que: $f_{i}=$ coeficiente de neutralização de escala do indicador $i ; N=$ quantidade de projetos analisados com indicadores positivos.

O segundo estágio do modelo é mostrado a seguir (Equações 10, 11, 12, 13, 14, 15, 16).

$$
\begin{aligned}
& \max Z=\sum_{i=1}^{P} f_{1} w_{1} x_{i} I L_{i}-f_{2} w_{2} x_{i} P B D_{i}- \\
& f_{3 w_{3} x_{i} G A F T i}
\end{aligned}
$$

sujeito a:

$$
\begin{aligned}
& -x_{32}-x_{34}+x_{42} \leq 1 \quad \text { (11) (restrição de dependência) } \\
& \sum_{j=1}^{M} \sum_{i=1}^{\mathrm{G}} x_{j i} \leq 1 \quad \text { (12) (restrição de mútua excludência) } \\
& \sum_{i=1}^{P} x_{i} D I_{i} \leq O R C \quad \text { (13) (restrição de orçamento) } \\
& \left.\right|_{i=1} ^{P} x_{i} M T I R_{i} \geq x_{i} T M A_{i} \quad \text { (14) (restrição de rentabilidade) } \\
& \left.\right|_{i=1} ^{P} x_{i} I L i>1 \quad \text { (15) (restrição de rentabilidade) } \\
& \left.\right|_{i=1} ^{P} x_{i} P B D_{i} \leq x_{i} V U_{i} \quad \text { (16) (restrição de liquidez) } \\
& \mathrm{x}_{\mathrm{i}} \in\{0,1\}
\end{aligned}
$$

em que: $I L_{i}=$ índice de lucratividade do projeto $i ; M_{T} R_{i}=$ taxa interna de retorno modificada do projeto $i ; P B D_{i}=$ payback descontado do projeto $i ; G A_{F T i}=$ grau de alavancagem combinado do projeto $i ; T M A i=$ taxa mínima de atratividade do 
Tabela 2. Projetos analisados e seus respectivos indicadores de rentabilidade, risco e liquidez.

\begin{tabular}{|c|c|c|c|c|c|c|c|c|c|}
\hline Grupo & Projeto & $\begin{array}{c}\text { DI } \\
(\mathbf{R} \$)\end{array}$ & $\begin{array}{c}T M A \\
(\% \text { aa) }\end{array}$ & $\begin{array}{c}\mathbf{N} \\
\text { (anos) }\end{array}$ & $\begin{array}{l}V P L \\
(\mathrm{R} \$)\end{array}$ & $\begin{array}{l}I L \\
(\%) \\
\end{array}$ & $\begin{array}{c}M^{M T I R^{3}} \\
(\%)\end{array}$ & $\begin{array}{c}P B D \\
\text { (anos) }\end{array}$ & $\begin{array}{c}G A_{\mathrm{FT}} \\
(\%)\end{array}$ \\
\hline \multirow{2}{*}{ A } & 1 & 1.000 & 10 & 4 & 39 & 3,91 & 11,06 & 2,90 & 15,57 \\
\hline & 2 & 1.000 & 10 & 4 & 53 & 5,35 & 11,44 & 4,70 & 19,32 \\
\hline \multirow{2}{*}{ B } & 3 & 1.000 & 12 & 4 & 58 & 5,80 & 13,59 & 4,60 & 20,06 \\
\hline & 4 & 1.000 & 12 & 4 & 39 & 3,99 & 13,10 & 3,70 & 15,88 \\
\hline \multirow{2}{*}{ C } & 5 & 22.000 & 12 & 6 & 3.860 & 17,55 & 15,06 & 5,30 & 33,58 \\
\hline & 6 & 17.500 & 12 & 6 & 3.057 & 17,47 & 15,05 & 5,30 & 33,49 \\
\hline \multirow{2}{*}{ D } & 7 & 10.000 & 12 & 5 & 814 & 8,14 & 13,77 & 5,10 & 22,41 \\
\hline & 8 & 25.000 & 12 & 5 & 1.675 & 6,70 & 13,46 & 5,10 & 20,77 \\
\hline \multirow{2}{*}{ E } & 9 & 300.000 & 9 & 5 & -43.883 & $-14,43$ & 5,66 & 20,00 & 8,29 \\
\hline & 10 & 120.000 & 9 & 5 & 253.406 & 211,17 & 36,78 & 2,40 & 250,13 \\
\hline \multirow{2}{*}{ F } & 11 & 68.000 & 10 & 10 & 84.385 & 124,10 & 19,24 & 4,20 & 156,74 \\
\hline & 12 & 28.000 & 10 & 5 & 44.783 & 159,94 & 33,16 & 2,70 & 193,09 \\
\hline \multirow{7}{*}{ G } & 13 & 5.000 & 8 & 4 & 701 & 14,03 & 11,60 & 4,60 & 28,13 \\
\hline & 14 & 10.000 & 8 & 3 & 970 & 9,70 & 11,39 & 3,80 & 22,59 \\
\hline & 15 & 10.000 & 8 & 4 & 3.248 & 32,49 & 15,87 & 3,80 & 48,29 \\
\hline & 16 & 12.000 & 8 & 3 & 885 & 7,38 & 10,59 & 3,70 & 19,80 \\
\hline & 17 & 8.000 & 8 & 2 & 2.699 & 33,74 & 24,90 & 2,50 & 48,71 \\
\hline & 18 & 5.000 & 8 & 2 & -216 & $-4,32$ & 5,64 & 10,00 & 6,64 \\
\hline & 19 & 6.000 & 8 & 4 & 2.153 & 35,89 & 16,60 & 3,90 & 52,31 \\
\hline \multirow{2}{*}{$\mathrm{H}$} & 20 & 100 & 10 & 2 & 38 & 38,84 & 29,61 & 2,40 & 54,75 \\
\hline & 21 & 80 & 10 & 2 & 32 & 41,01 & 30,62 & 2,40 & 57,17 \\
\hline \multirow{2}{*}{ I } & 22 & 100 & 10 & 2 & 17 & 17,36 & 19,16 & 2,80 & 31,22 \\
\hline & 23 & 100 & 10 & 2 & 16 & 16,74 & 18,85 & 2,40 & 29,81 \\
\hline \multirow{3}{*}{$\mathrm{J}$} & 24 & 480 & 9 & 7 & 170 & 35,46 & 13,83 & 5,90 & 53,90 \\
\hline & 25 & 620 & 9 & 7 & 92 & 14,97 & 11,19 & 6,80 & 30,59 \\
\hline & 26 & 750 & 9 & 7 & 192 & 25,60 & 12,61 & 6,30 & 42,67 \\
\hline \multirow{3}{*}{$\mathrm{K}$} & 27 & 10 & 10 & 2 & 21 & 214,05 & 94,94 & 0,60 & 248,97 \\
\hline & 28 & 5 & 10 & 2 & 16 & 321,49 & 125,83 & 2,00 & 371,05 \\
\hline & 29 & 5 & 10 & 2 & 11 & 238,84 & 102,48 & 2,00 & 278,52 \\
\hline \multirow{2}{*}{$\mathrm{L}$} & 30 & 5.000 & 10 & 5 & 1.338 & 26,76 & 15,34 & 4,50 & 42,93 \\
\hline & 31 & 8.000 & 10 & 10 & 1.794 & 22,43 & 12,35 & 6,90 & 40,26 \\
\hline \multirow{4}{*}{ M } & 32 & 1.500 & 10 & 5 & -610 & $-40,68$ & 2,16 & 10,00 & 35,37 \\
\hline & 33 & 1.500 & 10 & 5 & 766 & 51,07 & 19,46 & 5,20 & 72,63 \\
\hline & 34 & 1.500 & 10 & 5 & 796 & 53,09 & 19,78 & 5,20 & 74,33 \\
\hline & 35 & 1.500 & 10 & 5 & 779 & 51,95 & 19,60 & 4,40 & 72,06 \\
\hline \multirow{4}{*}{$\mathrm{N}$} & 36 & 85.000 & 20 & 4 & 18.549 & 21,82 & 26,07 & 3,90 & 38,92 \\
\hline & 37 & 150.000 & 20 & 4 & 51.921 & 34,61 & 29,26 & 3,60 & 53,51 \\
\hline & 38 & 250.000 & 20 & 4 & 87.577 & 35,03 & 29,36 & 4,50 & 58,03 \\
\hline & 39 & 378.000 & 20 & 4 & 19.337 & 5,12 & 21,51 & 4,10 & 18,52 \\
\hline \multirow{2}{*}{$\mathrm{O}$} & 40 & 100.000 & 10 & 5 & 84.337 & 84,34 & 16,94 & 5,2 & 111,19 \\
\hline & 41 & 70.000 & 10 & 5 & 52.891 & 75,56 & 16,37 & 5,5 & 101,13 \\
\hline \multirow{4}{*}{$\mathrm{P}$} & 42 & 80.000 & 10 & 5 & -9.339 & $-11,67$ & 7,30 & 10,00 & 5,41 \\
\hline & 43 & 20.000 & 15 & 7 & 2.399 & 12,00 & 16,88 & 6,50 & 29,07 \\
\hline & 44 & 500 & 20 & 10 & 128 & 25,70 & 22,78 & 7,00 & 47,23 \\
\hline & 45 & 200 & 20 & 10 & 219 & 109,62 & 29,22 & 3,82 & 145,39 \\
\hline Total & 45 & 1.805 .450 & & & 672.213 & 2.271 & & & 3.160 \\
\hline mín. & & 5 & 8 & 2 & -43.883 & $-40,68$ & 2,16 & 0,60 & 5,41 \\
\hline $\max$. & & 378.000 & 20 & 10 & 253.406 & 321,49 & 125,83 & 20,00 & 371,05 \\
\hline
\end{tabular}

Fonte: Elaborado pelo autor. Fonte: Abensur (2009), Brigham, Gapenski e Ehrhardt (2001), Hirschfeld (2009) e

Samanez (2009). ${ }^{3}$ Calculada pelo autor. Esses valores não vêm das obras citadas. 
projeto $i ; f i=$ coeficiente de neutralização de escala proveniente do primeiro estágio; $w=$ peso de cada componente da função objetivo; $p=$ conjunto de projetos analisados, $p \in\{1,2,3, \ldots ., P\} ; m=$ conjunto de projetos mutuamente excludentes, $m \in\{0,1,2,3, \ldots M\}$; $g=$ quantidade de projetos por grupo mutuamente excludente, $g \in\{1,2,3, \ldots ., G\} ; D I_{i}=$ desembolso inicial do projeto $i ; O R C=$ orçamento total disponível para os projetos; $V U_{i}=$ vida útil do projeto $i ; x_{i}=$ projeto $i$ participa da solução ótima.

\section{Resultados}

A relação dos projetos analisados e seus respectivos indicadores de desempenho são apresentados na Tabela 2. Os exemplos foram coletados de: Abensur (2009), Brigham, Gapenski e Ehrhardt (2001), Hirschfeld (2009) e Samanez (2009). Todos os grupos são independentes entre si. Os projetos dos grupos $A$ a $O$ são mutuamente excludentes. Os projetos do grupo $P$ são independentes entre si e independentes em relação a todos os outros. Os projetos 7-8; 11-12; 13-14; 15-17; 20-21; 24-26; 27-28; 30-31 e 36-39 apresentam, ao menos, dois indicadores contraditórios de comparação. O projeto 42 é dependente dos projetos 32 e 34 . Há um limite orçamentário arbitrariamente definido de $\mathrm{R} \$ 452.000,00$.

A Tabela 3 mostra as seleções obtidas pela aplicação do modelo matemático proposto com a função multiobjetivo e com a função mono-objetivo/IL tradicional. Conforme esperado, os projetos 9, 18, 32 e 42 não aparecem na solução final de nenhum dos métodos, pois apresentam VPL negativo. Também, conforme esperado, a seleção oferecida pelo modelo multiobjetivo é menor do que a do método tradicional, pois há mais critérios de avaliação. Parte da diferença entre as duas seleções pode ser atribuída à parcela de risco que contrabalanceou as parcelas de rentabilidade e ao $P B D$ muito próximo ou igual à vida útil dos projetos. Estas características não seriam percebidas numa função mono-objetivo.

Tabela 3. Projetos selecionados.

\begin{tabular}{|c|c|c|}
\hline Item & $\begin{array}{l}\text { Portfolio } \\
\text { proposto }\end{array}$ & $\begin{array}{c}\text { Portfolio } \\
\text { IL }\end{array}$ \\
\hline $\mathrm{n}^{\mathrm{o}}$ de projetos & 5 & 14 \\
\hline \multirow{3}{*}{ Projetos } & \multirow{3}{*}{$10-12-28-35-45$} & $1-4-5-10-12-19$ \\
\hline & & $24-28-30-35-37$ \\
\hline & & $43-44-45$ \\
\hline Investimento & $\mathrm{R} \$ 149.705$ & $R \$ 355.685$ \\
\hline$I L$ & 854,17 & 1050,04 \\
\hline$G A F T$ & 1031,72 & 1375,70 \\
\hline PBDmax & 4,4 anos & 7,0 anos \\
\hline
\end{tabular}

Fonte: Elaborado pelo autor.
A seleção mono-objetivo/IL incorpora projetos com baixa liquidez $(4,5,19,24,30,37,43)$ e com relação rentabilidade/risco $\left(I L / G A_{F T}\right)$ mais desfavorável $(4,5,43,44)$ podendo ser considerada, portanto, inferior à seleção do modelo proposto. Há uma economia de $\mathrm{R} \$ 205.980,00$ que pode ser direcionada para outras importantes atividades da empresa como reforço de caixa ou distribuição de dividendos.

O modelo proposto contribui na escolha dos projetos mesmo quando há indicadores contraditórios, pois há mais critérios de desempate ao longo da análise de todos os componentes da função multiobjetivo. $\mathrm{O}$ modelo também pode ser ajustado para favorecer algum critério conforme o peso atribuído na função objetivo. A Figura 1 apresenta um fragmento da planilha do Excel em que foram realizadas as simulações.

A Tabela 4 oferece a comparação entre os projetos selecionados pelo método proposto e pelos métodos tradicionais mono-objetivos (IL/VPL/PBD), caso cada grupo fosse avaliado separadamente. Em relação ao $I L$, houve nove seleções coincidentes e seis divergentes num total de quinze grupos analisados. Fica evidente a superioridade de seleção pelo método proposto. Os grupos $D, H, I$ e $O$ não apresentam projetos selecionados pelo método proposto porque violam a restrição de liquidez $(P B D<$ vida útil), mas são selecionados pelo $I L$.

Em relação ao método do VPL, houve seis seleções coincidentes e nove divergentes. Novamente, os grupos $D, H, I$ e $O$ que violam a restrição de liquidez são selecionados pelo $V P L$. As divergências apontadas pelo método proposto nos grupos $B$ e $M$, na comparação com o $I L$, e nos grupos $B, G, J, K$ e $M$, na comparação

Tabela 4. Projetos selecionados nos grupos mutuamente excludentes.

\begin{tabular}{ccccc}
\hline Grupo & $\begin{array}{c}\text { Método } \\
\text { proposto }\end{array}$ & $\begin{array}{c}\text { Método } \\
\boldsymbol{I L}\end{array}$ & $\begin{array}{c}\text { Método } \\
\boldsymbol{V} \boldsymbol{P L}\end{array}$ & $\begin{array}{c}\text { Método } \\
\boldsymbol{P} \boldsymbol{B D} \boldsymbol{~}\end{array}$ \\
\hline A & 2 & 2 & 2 & 1 \\
\hline B & 4 & 3 & 3 & 4 \\
\hline C & 5 & 5 & 5 & 5 ou 6 \\
\hline D & - & 7 & 8 & - \\
\hline E & 10 & 10 & 10 & 10 \\
\hline F & 12 & 12 & 12 & 12 \\
\hline G & 19 & 19 & 15 & 17 \\
\hline H & - & 21 & 20 & - \\
\hline I & - & 22 & 22 & - \\
\hline J & 24 & 24 & 26 & 24 \\
\hline K & 28 & 28 & 27 & 27 \\
\hline L & 30 & 30 & 30 & 30 \\
\hline M & 35 & 34 & 34 & 35 \\
\hline N & 38 & 38 & 38 & 37 \\
\hline O & - & 40 & 40 & - \\
\hline
\end{tabular}

Fonte: Elaborado pelo autor. 


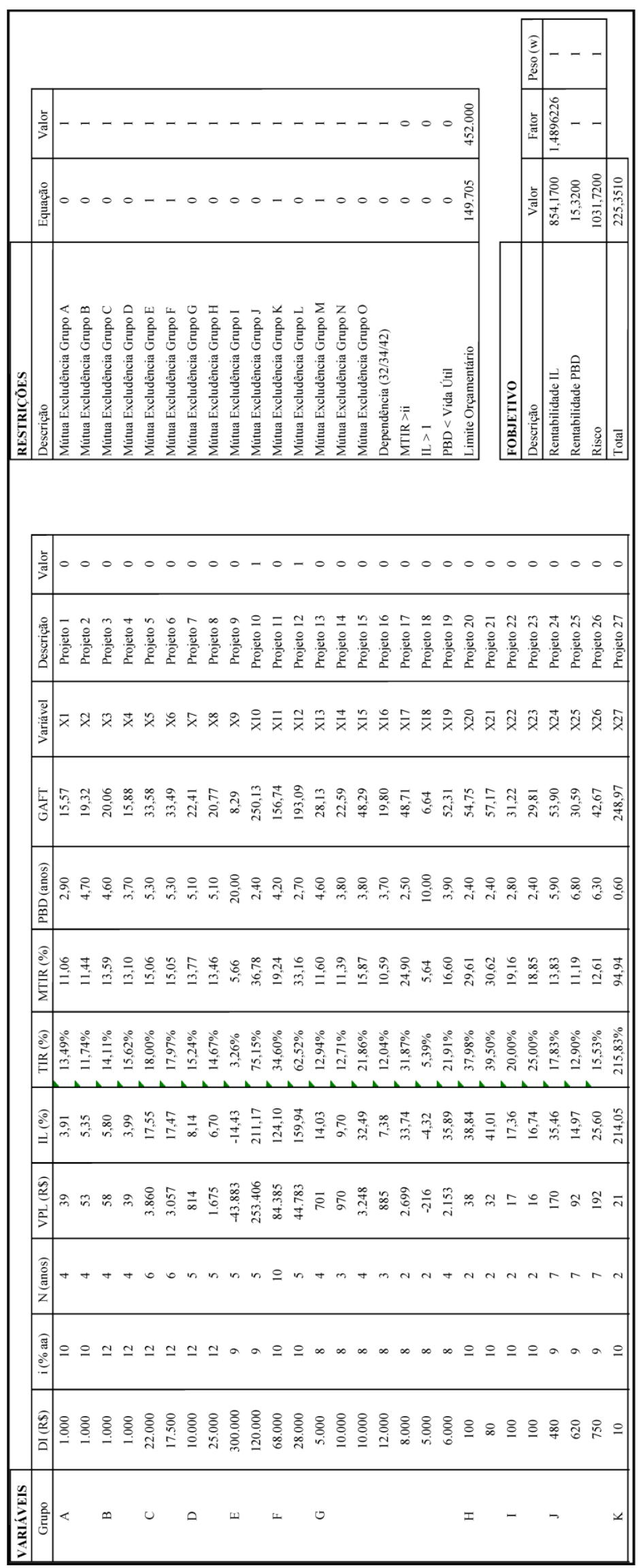


Tabela 5. Projetos selecionados por diferentes modelos de otimização.

\begin{tabular}{lcc}
\hline \multicolumn{1}{c}{ Item } & $\begin{array}{c}\text { Método } \\
\text { proposto }\end{array}$ & $\begin{array}{c}\text { Programação } \\
\text { de metas }\end{array}$ \\
\hline $\mathrm{n}^{\text {o }}$ de projetos & 5 & 9 \\
\hline Projetos & $10-12-28-35-45$ & $1-4-6-15-25$ \\
\cline { 2 - 3 } & $\mathrm{R} \$ 149.705$ & $\mathrm{R} \$ 1-36-43-44$ \\
\hline Investimento & 854,17 & 154,620 \\
\hline$I L$ & 1031,72 & 299,30 \\
\hline$G A_{\mathrm{FT}}$ & 4,4 anos & 7,0 anos \\
\hline PBDmax & & \\
\hline
\end{tabular}

Fonte: Elaborado pelo autor.

com o VPL, são consequência da influência dos indicadores de risco e liquidez da função multiobjetivo.

A comparação com o método $P B D$ mostrou dez coincidências e cinco divergências. A maioria das coincidências concentra-se nos grupos que violam a restrição de liquidez $(D, H, I, O)$. Conforme descrito na seção 2.3, o método $P B D$ privilegia os projetos de retorno mais rápido, mas, não necessariamente, os mais rentáveis como pode ser notado nas seleções feitas nos grupos $A, G, K$ e $N$.

Uma vez feitas as comparações com os métodos de análise de investimentos tradicionais, efetuou-se a comparação dos resultados obtidos pelo emprego de diferentes métodos de busca. A Tabela 5 apresenta os portfolios formados pelo método proposto e pela programação de metas, ambos usando os fatores de neutralização de escala.

A programação de metas visa alcançar uma solução de compromisso factível que minimize os desvios em relação aos objetivos estabelecidos. No caso analisado, as metas são: (i) maximizar a rentabilidade $(I L)$; (ii) minimizar o retorno $(P B D)$; e (iii) minimizar o risco $\left(G A_{F T}\right)$. A meta (i) é conflitante com as metas (ii) e (iii). Maximizar a rentabilidade induz a selecionar o maior número possível de projetos, enquanto, que as metas de minimização de retorno e risco induzem a não investir em nenhum projeto.

O portfolio da programação de metas mostra uma necessidade de investimento $4 \%(\mathrm{R} \$ 6.085,00)$ inferior ao do modelo proposto e com menor risco. No entanto, o portfolio formado apresenta mais projetos, é bem menos rentável e com prazo de retorno mais desfavorável. Novamente, esta seleção pode ser considerada inferior à obtida pelo modelo proposto.

\section{Considerações finais}

Nenhum método tradicional de análise de investimentos atende, isoladamente, a todos os critérios para uma decisiva seleção de projetos de diferentes naturezas. Isto restringe o uso de funções mono-objetivas como forma de otimização para o processo de orçamento de capital.
A combinação e integração dos métodos mais recomendados de análise de rentabilidade, liquidez e risco com técnicas de otimização aumentam as chances de uma seleção superior de projetos. Foi desenvolvido um modelo de otimização em dois estágios que permite a incorporação de indicadores de desempenho de diferentes naturezas e intensidades. Além disso, conforme o método de otimização usado neste estudo, há a garantia de solução ótima para a seleção obtida.

O modelo proposto está alinhado com os conceitos da moderna teoria financeira, da gestão de risco e com as práticas nacionais e internacionais. Foi criada uma medida de risco bidimensional $\left(G A_{F T}\right)$ a partir dos dados disponíveis para análise. A introdução desta componente de risco trouxe equilíbrio ao processo de seleção indo além da análise tradicional focada exclusivamente em rentabilidade. Uma interessante linha de pesquisa seria a avaliação de outras dimensões para a análise do risco do projeto.

A introdução de um indicador de liquidez expande as possibilidades de seleção além da tradicional dualidade retorno vs. risco. Tacitamente, os investidores incorporam a noção de liquidez nas suas decisões, ou seja, em igualdade de condições nos outros indicadores, seriam escolhidos os projetos que oferecessem retornos mais rápidos. Várias pesquisas mostram que a ausência de custos de transação e liquidez (ou falta de liquidez) resulta na formação de portfolios ineficientes (AMIHUD; MENDELSON, 1991; ARNOTT; WAGNER, 1990; JANA; ROY; MAZUNDER, 2009).

O modelo proposto e seus resultados não devem ser analisados individualmente, mas como parte de um processo de orçamento de capital. Projetos considerados de alto risco e que, portanto, podem prejudicar a liquidez (caixa) da empresa devem ser separados para uma análise individual e diferenciada e não fariam parte da relação de projetos candidatos para avaliação do modelo proposto. Experimentos industriais conduzidos por simulação por computador e/ou por protótipos físicos são exemplos praticados, entre outros, pelas indústrias automobilística, aeroespacial e petrolífera (THOMKE, 1998).

Conforme apresentado na seção 1, o modelo foi testado sobre exemplos teóricos oferecidos pela literatura sem prejuízo de qualidade sobre os resultados, pois representam situações hipotéticas factíveis. Seria interessante testá-lo sobre situações reais (estudos de caso) para avaliar a aderência dos resultados com as opiniões dos gestores.

Outra linha de pesquisa seria a avaliação dos pesos e componentes usados na função objetivo-proposta por meio da adoção de métodos de apoio à decisão multicritério como, por exemplo, Analytic Hierarchy Process (AHP), Multi-Atribute Utility Theory (MAUT), Elimination et Choix Traduisant La 
Realité (ELECTRE) e Measuring Attractiveness by a Categorical Based Evolution Techinique (Macbeth) (SONCINI, 2008; SOUZA; KLIEMANN NETO; FILOMENA, 2010).

\section{Referências}

ABENSUR, E. O. Finanças Corporativas: fundamentos, práticas brasileiras e aplicações em planilha eletrônica e calculadora financeira. São Paulo: Scortecci, 2009.

AMIHUD Y.; MENDELSON, H. Liquidity, Asset Prices and Financial Policy. Financial Analysts Journal, v. 47, n. 6, p. 56-66, 1991. http://dx.doi.org/10.2469/ faj.v47.n6.56

ARNOTT, R. D.; WAGNER, W. H. The Measurement and Control of Trading Costs. Financial Analysts Journal, v. 46 , n. 6 , p. $73-80,1990$. http://dx.doi.org/10.2469/ faj.v46.n6.73

ASSOCIAÇÃO BRASILEIRA DE NORMAS TÉCNICAS - ABNT. NBR ISO 31000: Gestão de riscos, princípios e diretrizes. Rio de Janeiro: ABNT, 2009.

BANCO CENTRAL DO BRASIL - BCB. SGS - Sistema Gerenciador de Séries Temporais. Disponível em: $<$ https://www3.bcb.gov.br/sgspub/consultarvalores/ telaCvsSelecionarSeries.paint>. Acesso em: 09 out. 2011.

BREALEY, R. A.; MYERS, S. C.; ALLEN, F. Princípios de Finanças Corporativas. São Paulo: McGraw-Hill, 2008. BRIGHAM, E. F.; GAPENSKI, L. C.; EHRHARDT, M. C. Administração Financeira: Teoria e Prática. São Paulo: Atlas, 2001.

CASAROTTO FILHO, N.; KOPITTKE, B. H. Análise de Investimentos: matemática financeira, engenharia econômica, tomada de decisão, estratégia empresarial. São Paulo: Atlas, 2010.

COLIN, E. C. Pesquisa Operacional: 170 Aplicações em Estratégia, Finanças, Logística, Produção, Marketing e Venda. Rio de Janeiro: LTC, 2007.

COMPANHIA VALE DO RIO DOCE - CVRD. Investimentos. Disponível em: http://www.vale.com/ pt-br/investidores/>. Acesso em: 13 jun. 2011a.

COMPANHIA VALE DO RIO DOCE - CVRD. Resultados e Informações Financeiras. Disponível em: <http:// www.vale.com/pt-br/investidores/>. Acesso em: 13 jun. 2011b.

COOPER, R.; EDGETT, S.; KLEINSCHMIDT, E. New Product Management: Practices and Performance. Journal of Product Innovation Management, v. 16, n. 4, p. 333-351, 1999. http://dx.doi.org/10.1016/ S0737-6782(99)00005-3

COSTA NETO, P. L. O. Estatística. São Paulo: Edgard Blücher, 2000.

EID JÚNIOR, W. Custo e estrutura de capital: o comportamento das empresas brasileiras. Revista de Administração de Empresas, v. 36, n. 4, p. 51-59, 1996.
GITMAN, L. J.; MADURA, J. Administração Financeira: uma Abordagem Gerencial. São Paulo: Pearson Addison Wesley, 2003.

HILLIER, F. S.; LIEBERMAN, G. J. Introdução à Pesquisa Operacional. Rio de Janeiro: Campus, 1988.

HIRSCHFELD, H. Engenharia Econômica e Análise de Custos: aplicações práticas para economistas, engenheiros, analistas de investimentos e administradores. São Paulo: Atlas, 2009.

JANA, P.; ROY, T. K.; MAZUNDER, S. K. Multi-objective Possibilistic Model for Portfolio Selection with Transaction Cost. Journal of Computational and Applied Mathematics, v. 228, n. 1, p. 188-196, 2009. http://dx.doi.org/10.1016/j.cam.2008.09.008

JORION, P. Value at Risk: A Nova Fonte de Referência para a Gestão do Risco Financeiro. São Paulo: Bolsa de Mercadoria \& Futuros, 2003.

LACHTERMACHER, G. Pesquisa Operacional na Tomada de Decisão. Rio de Janeiro: Campus, 2002.

MACEDO, M. A. S. Seleção de Projetos de Investimento: uma proposta de modelagem apoiada em Programação Multi-Objetivo. In: ENCONTRO BRASILEIRO DE FINANÇAS, 5., 2005, São Paulo. Anais... São Paulo: Mackenzie, 2005.

MARKOWITZ, H. Portfolio Selection. Journal of Finance, v. 7, n. 1, p. 77-91, 1952.

PADOVANI, M.; CARVALHO, M. M.; MUSCAT, A. R. N. Seleção e Alocação de Recursos em Portfólio de Projetos: Estudo de Caso no Setor Químico. Gestão \& Produção, v. 17, n. 1, p. 157-180, 2010. http://dx.doi. org/10.1590/S0104-530X2010000100013

ROSS, S. A.; WESTERFIELD, R. W.; JAFFE, J. F. Administração Financeira. São Paulo: Atlas, 2002.

SAMANEZ, C. P. Engenharia Econômica. São Paulo: Pearson Prentice Hall, 2009.

SHARPE, W. Capital Asset Prices: A Theory of Market Equilibrium Under Conditions of Risk. Journal of Finance, v. 19, n. 3, p. 425-442, 1964.

SONCINI, P. Modelagem multicriterial para Análise de Projetos de Investimento - o caso de uma distribuidora de energia elétrica. 2008. 152 F. Dissertação (Mestrado em Engenharia)-Universidade Federal do Rio Grande do Sul, Porto Alegre, 2008.

SOUZA, J. S.; KLIEMANN NETO, F. J.; FILOMENA, T. P. Definição de portfolio de investimentos em uma empresa usando Análise Multicriterial. Revista Produção On-Line, v. 10, n.1, p. 168-196, 2010.

TAHA, H. A. Pesquisa Operacional. São Paulo: Pearson Prentice Hall, 2008.

THOMKE, S. H. Simulation, Learning and R\&D Performance: evidence from automotive development. Research Policy, v. 27, p. 55-74, 1998. http://dx.doi. org/10.1016/S0048-7333(98)00024-9 